\title{
Splitting hairs and other intermediate filaments
}

\section{from Peter M. Steinert and Alasdair C. Steven}

EVEN though keratinized tissues rich in intermediate filaments were possibly the first biological substances to be probed by $\mathrm{X}$-ray diffraction techniques (ref. 1), solving their structure remains a plum to be picked. These filaments, $10-15 \mathrm{~nm}$ in diameter, form one of the three major cytoskeletal networks in eukaryotic cells. They differ from the other two, F-actin and microtubules, in several important respects. For example, unlike the other two, intermediate filaments are composed of $\alpha$ helix rich fibrous protein subunits, and their distributions and state of assembly in cells seem to be governed by quite different rules. Their precise function(s) in cells remain obscure, so an understanding of the details of their structure is an important first step in elucidating their cellular functions. A flurry of recent papers describing the amino-acid sequences of around 30 subunits suggests that the broad principles of intermediate filament structure will soon be understood.

All the filaments have been found to contain a central $\alpha$-helical 'rod' domain of conserved secondary structure, flanked by end domains of variable size and chemical character. The rod domains possess four discrete segments of precisely conserved size that can form coiled-coils, termed $1 \mathrm{~A}, 1 \mathrm{~B}, 2 \mathrm{~A}$ and $2 \mathrm{~B}$, interspersed by regions that cannot form coiled-coils ( $a$ in figure). The rod domain sequences conform to several distinct types: the $10-15$ acidic keratins (Type I); the $10-15$ neutral-basic keratins (Type II); vimentin, desmin and glial fibrillary acidic protein (Type III); and neurofilament proteins (possibly comprising at least one other type ${ }^{2}$ ). Convincing evidence now shows that pairs of subunits align in parallel and in axial register to form two-chain coiled-coil molecules ( $b$ in figure $)^{2}$ but it is not known how these molecules combine to generate higher orders of intermediate filament structure. Observations using the scanning transmission electron microscope $^{3}$ suggest that most filaments,

\section{Years Ago}

MANY interesting excursions have been arranged for the Aberdeen meeting of the British Association. One of them will, of course, be to the great granite quarries in the neighbourhood of Aberdeen. Her Majesty has invited 150 of the members to Balmoral, where they will be shown over the grounds and have lunch. It is not to be expected that the Queen will personally receive all the members, though it is possible that a few representative men of science may be presented to Her Majesty.

From Nature 32 400, 27 August 1885. irrespective of their subunit composition, contain about 16 molecules in crosssection. These and other data have also contributed to the emerging concept that the rod domains form the cores of intermediate filaments and that the interactions of these domains are primarily responsible for defining filament structure. The end domains are located on the periphery of this core and define the functions of different filaments in cells ${ }^{2,3}$.

At the immediately supramolecular level, several lines of evidence invoke a dimeric association of molecules in a fourchain oligomeric complex ${ }^{4,5}$. It is plausible that such an association should be based

a Subunit model

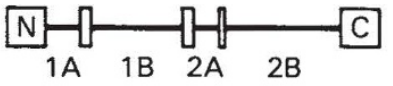

cPossible models for four-chain complex
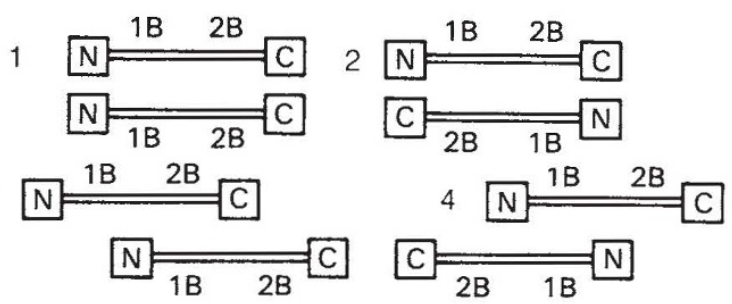

on alignment of the long (1B and $2 \mathrm{~B}$ ) coiled-coil segments ${ }^{\text {; }}$; if so, there are essentially only five possibilities ( $c$ in figure). Models 1 and 2 predict the segments to be about $48 \mathrm{~nm}$ long and the others predict them to be about $70 \mathrm{~nm}$.

Two recent studies have cast some light on these alternatives. Using quick-freeze deep-etching electron microscope techniques, Ip et al. ${ }^{6}$ have characterized a $48 \mathrm{~nm}$ particle as the tetrameric building block for vimentin. This is consistent with models 1 or 2 , but cannot distinguish between them as the differences depend on the polarity of the molecules. But, by using a monoclonal antibody against region $2 \mathrm{~B}$ of the desmin rod, Geisler et al. ${ }^{7}$ seem to have answered this question. They found dumbbell-shaped structures averaging 48 $\mathrm{nm}$ in length, denoting antibody binding at both ends of a similar tetrameric desmin complex and compatible only with model 2. However, in other studies on isolated $\alpha$-helix-enriched particles obtained from proteolysed keratin intermediate filaments, $1 \mathrm{~B}$ tetramer particles were obtained $^{8.9}$ which could only have derived from models 1 or 4 and not from model 2 . Under modified salt conditions, Ip et al. ${ }^{6}$ visualized larger 70-nm-long vimentin
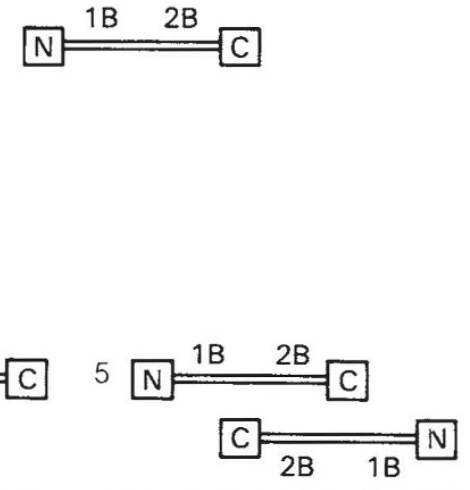

particles, but interpreted them not as models $3-5$, but as staggered pairs of fourchain complexes. Again, the complexes might be parallel or antiparallel, but their neighbouring constituent twochain molecules should nevertheless comply with models shown in $c$.

The ingenuity of these experiments notwithstanding, their apparent incompatibility poses a problem: which data are correct? Or is every investigator right? Assuming that keratin and Type III intermediate filaments do share a common structure, there may be at least one way to reconcile this paradox. Conceivably, there may be multiple modes of intermolecular associations in fully assembled filaments, so that more than one of the models in $c$ may be applicable. Then, disassembly or reassembly of intermediate filaments under conditions of varying salt or $p \mathrm{H}$ might selectively weaken different sets of interactions and split the same structure into different four-chain complexes. This idea could be tested by the

\section{bTwo-chain molecule}

experimental approaches described here.

Taken together, recent reports make it likely that neighbouring pairs of two-chain coiled-coil molecules in intermediate filaments are antiparallel, meaning that the filaments are non-polar structures. Thus, unlike the other mainstays of the cytoskeleton, F-actin and microtubules, intermediate filaments don't know whether they are coming or going. Further developments in this rapidly evolving field hopefully will extricate researchers from a similar dilemma.

1. Astbury, W.T. \& Street. A. Phil. Trans. R. Soc. A230, 75 (1931)

2. Steinert, P.M., Steven, A.C. \& Roop, D.R. Cell (in the press).

3. Steven, A.C., Hainfeld, J.T., Tr, B.L., Wall, J.S. \& Steinert, P.M. J. Cell Biol. 97, 1939 (1983).

. Crewther, W.G., Dowling, L.M., Steinert, P.M. \& Parry, D.A.D. Int. J. biol. Macromol. 5. 267 (1983).

6. Ip. W., Hartzer, M.K., Pang, Y.-Y. \& Robson, R.M. J. molec. Biol. (in the press).

. Geisler, N., Kaufmann, E. \& Webber, K. J. molec. Biol. 182, 173 (1985).

8. Woods, E.F., Inglis, A.S. Int. J. biol. Macromol. 6, 277 (1984)

9. Parry. D.A.D., Steven, A.C., Steinert, P.M. Biochem. biophys. Res. Commun. 127, 1012 (1985).

Peter M. Steinert is in the Dermatology Branch, $\mathrm{NCl}$, and Alasdair C. Steven is in the Laboratory of Cellular and Developmental Biology, NIADDK, National Institutes of Health, Bethesda, Maryland 20205, USA.
4. Geisler, N. \& Weber, K. EMBO J. 1, 1649 (1982). 